\title{
Review of: "Benefits and harms of Risperidone and Paliperidone for treatment of patients with schizophrenia or bipolar disorder: a meta-analysis involving individual participant data and clinical study reports"
}

\author{
Katrine Kveli Fjukstad
}

Potential competing interests: The author(s) declared that no potential competing interests exist.

This meta-analysis is largely well-written and well-conducted. The topic is of great importance. There are however some issues in the article that should be addressed.

1. The authors makes a statement regarding double diagnosis of bipolar disorder and schizophrenia with a reference to genetic similarities. A reference stating the prevalence of double diagnosis are missing.

2. Why were schizoaffective disorder not included?

3. The metabolism of risperidone and hence paliperidone should be elaborated to highlight the similarities and differences in these drugs.

4. The handling of missing data is a strength, but the amount of missing data should be stated some sort.

5. A table reporting demographic data and administration form would be of interest.

6. Risperidone Consta is not administrated intravenously. Please correct.

7. A precision regarding the chosen method of I squared statistics would be appreciated.

8. Recommend figure revision of figure 2 . Some data are in bold and the readability is poor. Suggest providing more information of included trials (ID, N, dose range, duration of trials). All figures should have a heading.

9. Strengths and weaknesses of this trial should be discussed in the discussion section.

10. The included trials had an large overweight of male subjects. This should be discussed as the gender differences in risperidone/paliperidone adverse effects can be substantial (prolonged exposure to D2 antagonists in women - amenorrea, osteoporosis, breast cancer).

11. The large dose range and the effect of administration forms are not sufficiently addressed in the discussion. Does any of the included trials have any information regarding adherence or serum concentrations of drugs?

12. The discussion section are largely politicizing. This can be compressed and still give important insights. There should be a more elaborate discussion regarding the actual findings. Any alternative explanations of the discrepancies between studies and whether they are more or less likely would increase the credibility. 
13. I have not reviewed the supplemental files as the doi were inactive or I were unable to open it due to fire walls. 